\title{
Brain imaging predictors and the international study to predict optimized treatment for depression: study protocol for a randomized controlled trial
}

Stuart M Grieve ${ }^{1,2,3,4^{*}}$, Mayuresh S Korgaonkar ${ }^{1,5}$, Amit Etkin ${ }^{6,7}$, Anthony Harris ${ }^{1,5}$, Stephen H Koslow ${ }^{8,9}$, Stephen Wisniewski ${ }^{7}$, Alan F Schatzberg ${ }^{6}$, Charles B Nemeroff ${ }^{8}$, Evian Gordon ${ }^{1,2}$ and Leanne M Williams ${ }^{1,5,6}$

\begin{abstract}
Background: Approximately 50\% of patients with major depressive disorder (MDD) do not respond optimally to antidepressant treatments. Given this is a large proportion of the patient population, pretreatment tests that predict which patients will respond to which types of treatment could save time, money and patient burden. Brain imaging offers a means to identify treatment predictors that are grounded in the neurobiology of the treatment and the pathophysiology of MDD.

Methods/Design: The international Study to Predict Optimized Treatment in Depression is a multi-center, parallel model, randomized clinical trial with an embedded imaging sub-study to identify such predictors. We focus on brain circuits implicated in major depressive disorder and its treatment. In the full trial, depressed participants are randomized to receive escitalopram, sertraline or venlafaxine-XR (open-label). They are assessed using standardized multiple clinical, cognitive-emotional behavioral, electroencephalographic and genetic measures at baseline and at eight weeks post-treatment. Overall, 2,016 depressed participants (18 to 65 years old) will enter the study, of whom a target of $10 \%$ will be recruited into the brain imaging sub-study (approximately 67 participants in each treatment arm) and 67 controls. The imaging sub-study is conducted at the University of Sydney and at Stanford University. Structural studies include high-resolution three-dimensional T1-weighted, diffusion tensor and T2/Proton Density scans. Functional studies include standardized functional magnetic resonance imaging (MRI) with three cognitive tasks (auditory oddball, a continuous performance task, and Go-NoGo) and two emotion tasks (unmasked conscious and masked non-conscious emotion processing tasks). After eight weeks of treatment, the functional MRI is repeated with the above tasks. We will establish the methods in the first 30 patients. Then we will identify predictors in the first half $(n=102)$, test the findings in the second half, and then extend the analyses to the total sample.
\end{abstract}

Trial registration: International Study to Predict Optimized Treatment - in Depression (iSPOT-D). ClinicalTrials.gov, NCT00693849.

Keywords: Major depressive disorder, Antidepressant treatments, Imaging, Biomarker, iSPOT-D

\footnotetext{
* Correspondence: sgrieve@med.usyd.edu.au

'The Brain Dynamics Centre, University of Sydney Medical School -

Westmead and Westmead Millennium Institute, Sydney, NSW 2145, Australia

${ }^{2}$ Brain Resource, Level 12, 235 Jones Street, Ultimo, Sydney, NSW 2007,

Australia and Suite 200, 1000 Sansome Street, San Francisco, CA 94111, USA

Full list of author information is available at the end of the article
} 


\section{Background}

Major depressive disorder (MDD) is a leading cause of morbidity, mortality and disability for individuals aged 15 to 44 years [1]. A substantial percentage, (approximately $50 \%$ ), of patients do not respond to antidepressant treatments and these individuals constitute a large proportion of the disease burden [2-5]. There is an urgent need for objective tests that can be obtained before initiating treatment to predict which patients will go on to show a clinical response to treatment, and to identify which type of treatment is best for the individual patient. Functional and structural brain imaging offers a means to identify treatment predictors, which are based upon the neurobiological mechanisms of these treatments, and the means to the pathophysiology of MDD and its subtypes.

The international Study to Predict Optimized Treatment for Depression (iSPOT-D) [6] is a large, international, multi-center, parallel model, clinical randomized trial which seeks to identify factors that predict treatment response to three common antidepressants. In iSPOT-D, we have combined a practical clinical trial design with the use of brain imaging to identify imaging predictors of treatment outcome in a real-world setting, along with other objective measures, such as electrophysiology and cognitive performance metrics. This paper presents the background, rationale and methodology for the imaging sub-study embedded within iSPOT-D.

Recent developments in neuroimaging have yielded powerful tools that enable quantification of both functional and static neural connectivity. These tools include diffusion tensor imaging (DTI), functional magnetic resonance imaging (fMRI), resting state fMRI, arterial spin labeling, positron emission tomography (PET) and high-resolution cortical mapping techniques that enable the automated, accurate localization of brain regions. Unfortunately, the majority of studies that have used these techniques to address treatment prediction in MDD have been underpowered, employing small cohorts (for example, $\mathrm{n}=20$ to 30 ), and they have typically focused upon a single imaging measure rather than seek convergence across multiple measures. Also, to date, no published study has been designed to test prediction across multiple antidepressants. However, a considerable body of data has examined the structural and functional differences that exist between the brains of depressed and non-depressed patients. These studies served to identify the brain circuits that represent viable targets for testing predictors of treatment outcome.

The rationale behind the use of three treatment arms in iSPOT-D is to identify what are the unique and common brain circuits that modulate response and remission to different types of anti-depressant medications, that is, selective serotonin reuptake inhibitors (SSRIs) versus selective norepinephrine reuptake inhibitors (SNRIs), or between the two different types of SSRIs. The receptor-level pharmacological actions of these medications may not be the best indicator of the mechanisms by which they lead to remission of depression, (which may take weeks, unlike receptor binding), and which is the primary focus of our analysis. For instance, sertraline and citalopram (the parent compounds for escitalopram) have been found to have differential effects in animals on hippocampal neuronal excitability [7], and are also known to differentially alter behavior in humans that are relevant to the brain circuits assessed in this study [8]. To date, there have been a few studies using small samples that examine the functional imaging predictors of response to individual antidepressants. These studies provide the basis for our rationale for the study. For example, using emotion activation tasks, depression has been associated with hyper-reactivity of amygdala circuits, which attenuates the following response to SSRIs [9-11]. The antidepressant sertraline has similarly been found to predict response and "normalize" amygdala hyperactivity and medial prefrontal hypo-activity, which characterize generalized social phobia, a disorder often comorbid with depression [12].

The need for a new mechanism-based approach to diagnosing and treating depression has been recognized in several initiatives. First, the research agenda for DSM-5 (the fifth edition of Diagnostic and Statistical Manual of Mental Disorders) emphasizes a need to translate neuroscience research findings into a new classification system for mental illnesses based on underlying brain circuitry [13]. Second, the Research Domain Criteria project - a National Institute of Mental Health initiative - aims to "develop, for research purposes, new ways of classifying mental disorders based on dimensions of observable behaviour and neurobiological measures" [14,15]. These objectives have also been touched upon by the recent Mayflower Action Group Initiative [16]. Imaging techniques, such as magnetic resonance imaging (MRI), provide a precise window into the circuits that play a role in the pathogenesis of depression and in the response to treatment, enabling the quantification of these circuits as potentially predictive "biomarkers". The National Institutes of Health biomarker working group defined a biomarker as: "a characteristic that is objectively measured and evaluated as an indicator of normal biological processes, pathogenic processes, or pharmacologic responses to a therapeutic intervention" [17]. There are currently no clinically useful biomarkers for predicting response to antidepressant medications [18], a shortfall that the imaging sub-study of the iSPOT-D is designed to address.

In this paper, we characterize the key features of the iSPOT-D imaging sub-study. These features include (i) a focus on the discovery of imaging biomarkers that are likely to be clinically useful in the treatment of depression; (ii) a large and representative sample to achieve generalizable results, including a large percentage of treatment-naive 
participants; (iii) a translational design involving three common, current treatment regimes to provide an understanding of response heterogeneity; (iv) a highly standardized approach across sites to maximize the power of the data; and (v) a multi-modality MRI-based approach that provides convergent evidence that highlights key changes that are present.

Specifically, the iSPOT-D imaging study will serve to answer the following key questions:

1. What pre-treatment aspects of brain circuitry predict and moderate responses to anti-depressants?

2. What aspects of brain circuitry improve following treatment? We expect partially distinct circuitry to be involved in predicting response and remission to different antidepressants.

3. Do these predictive aspects of brain circuitry overlap with the core imaging features that are seen in depression when we compare depressed patients to healthy controls? Drawing on the available evidence to date, it is expected that those circuits impaired in depression will also be the mechanism by which antidepressants exert their mechanism of action. Thus, we will also assess if depression-related impairments are "normalized" post-treatment.

4. Are there additional distinctive alterations that identify specific "subtypes" of depression?

5. Are there associations between the imaging measures and the other data available from the iSPOT-D protocol?

\section{Methods/Design}

\section{Study design}

The structure of the parent iSPOT-D trial has been previously described in detail [6]. Briefly, it is a multi-center, international, prospective, parallel model, open-label randomized clinical trial involving 2,016 MDD patients aged 18 to 65 years. There are three treatment arms (escitalopram, sertraline and venlafaxine-XR) and a control arm, each with 672 participants. The healthy control participants are studied only at baseline, while the depressed participants are studied both at baseline and at eight weeks. The two sites for the imaging sub-study are the Brain Dynamics Center, University of Sydney (Sydney) and the Department of Psychiatry and Behavioral Sciences, Stanford University (Stanford). A target of 10\% of iSPOT-D participants are to be recruited into the brain imaging sub-study with an overall target of approximately 200 participants with MDD (67 in each treatment arm) and 67 in the control group.

\section{Organizational structure/Acquisition sites}

The iSPOT-D Executive Committee for the trial oversees the overall study. Supervision of the study on a day-by -day basis is performed at the Global Coordinating
Center and Data Center (Sydney). Coordination of the study occurs via a Global Trial Coordinator and the executive management team. There are 20 global clinical sites involved in patient recruitment, though only two sites are involved in the imaging sub-study (Sydney and Stanford). The imaging-specific components of the substudy are overseen by the senior imaging scientist (MSK) and the head of the imaging unit (SMG) at the central imaging site (University of Sydney, Westmead Hospital). The imaging data obtained at the secondary imaging site (Stanford University) are monitored at both the point of acquisition and the central imaging facility.

\section{Study participants}

Details of the study inclusion-exclusion criteria are provided in Williams et al. [6]. In short, the primary diagnosis of nonpsychotic MDD is confirmed using the MiniInternational Neuropsychiatric Interview (MINI) [2,3], according to DSM-IV criteria, and a score $\geq 16$ on the 17-item Hamilton Rating Scale for Depression (HRSD17) [4]. All MDD participants are either antidepressant medication (ADM) naïve or, if they had been previously prescribed an ADM, had undergone a wash-out period of at least five half-lives. Healthy control participants are extensively screened for the absence of Axis I disorders (using the MINI) and for an $\mathrm{HRSD}_{17}$ score $<7$.

Imaging participants are selected by sequentially recruiting patients at the time of enrollment (Figure 1). Additional MRI-specific exclusion criteria not applied to the general patients include the standard safety criteria for MRI scanning: a cardiac pacing/defibrillator device, claustrophobia, the presence of a magnet-incompatible metal prosthesis. Demographic information for both the MDD and control groups in the first $15 \%$ of participants is summarized in Table 1.

\section{Treatment}

Participants were randomized to treatment using PhaseForward's ${ }^{\text {Tw }}$ validated, Web-based Interactive Response Technology. A blocked randomization procedure was undertaken centrally (block size: 12, across sites). Neither participants nor investigators/raters were blinded to treatment assignment. The participant's treating clinician (general practitioners and psychiatrists) prescribed ADMs adjusted dosage according to routine clinical practice.

\section{Ethical considerations}

The study is being conducted according to the principles of the Declaration of Helsinki 2008 and the International Conference on Harmonization guidelines. Local regulations were also observed: The Australian Therapeutic Goods Association [19] and the United States Food and Drug Administration Code of Federal Regulations [20]. Prior to enrollment, the study procedures are fully 


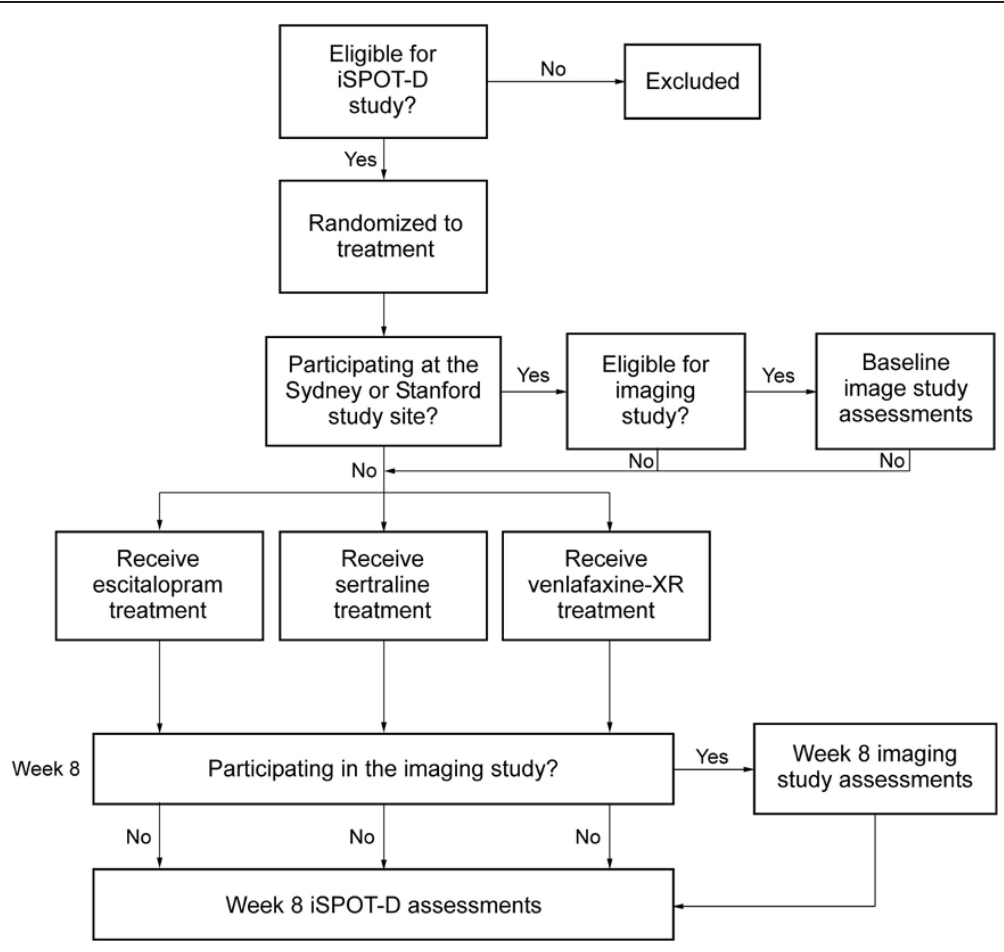

Figure 1 Trial flowchart.

explained to the study participant in accordance with the ethical guidelines of the institutional review board and written informed consent is obtained.

\section{MRI data acquisition}

MRI studies are performed on a 3.0 Tesla GE SignaTwinspeedHDxtmagnet system (GE Healthcare, Milwaukee, WI, USA) utilizing an 8-channel (Westmead) or a 3.0 Tesla GE Discovery MR750magnet system utilizing a 32-channel (Stanford) phased-array head coil. Specialized equipment is also used to facilitate the fMRI data collection (Resonance Technology, Los Angeles, CA, USA) and the same standardized software is used for the delivery of

Table 1 Demographics for the MDD and control groups from the first $15 \%$ of participants

\begin{tabular}{lcc}
\hline Measures & $\begin{array}{c}\text { Controls } \\
(\mathbf{n}=\mathbf{3 0})\end{array}$ & $\begin{array}{c}\text { MDD patients } \\
(\mathbf{n}=\mathbf{3 0})\end{array}$ \\
\hline Male/Females & $12 / 18$ & $12 / 18$ \\
Right-/Left-handed & $26 / 4$ & $26 / 4$ \\
& Mean \pm SD & Mean \pm SD \\
Age (years) & $35.7 \pm 14.1$ & $41.2 \pm 15.8$ \\
Education (years) & $15.1 \pm 2.8$ & $14.5 \pm 3.2$ \\
HRSD $_{17}$ & $1.3 \pm 1.4$ & $19.2 \pm 3.1^{\mathrm{a}}$
\end{tabular}

${ }^{a}$ significant difference with Control group at $P<0.001$, independent sample $t$-test.

HRSD $_{17}$ 17-item Hamilton Rating Scale for Depression; MDD, Major depressive disorder. functional stimulus (Stimsoft v1.2.1, Neuroscan, El Paso, TX). All imaging protocols were standardized and crosssite consistency established for data comparison. Table 2 shows cross-site validation of the $\mathrm{T} 1$ spoiled gradient recalled anatomical protocol using test-retest MRI scans collected on the same individual scanned at both the Sydney and Stanford magnetic resonance scanners. Table 3 presents a detailed summary of the acquisition parameters used. The imaging protocol takes approximately 1 hour and15 minutes to complete, including patient positioning. The protocol comprises both functional and structural MRI components. The full protocol is acquired at the initial visit; fMRI measures are replicated at the second time point only (imaging time: 45 minutes).

The structural dataset comprises three scans: a highresolution three-dimensional (3D) T1-weighted volume dataset (1 mm isotropic resolution; repetition time (TR) $8.3 \mathrm{~ms}$; echo time (TE) $3.2 \mathrm{~ms}$; inversion time (TI) $500 \mathrm{~ms})$, DTI data $(1.7 \times 1.7$ in-plane resolution; $2.5 \mathrm{~mm}$ slice thickness; TR 17,000 ms; TE $95 \mathrm{~ms}$ ) and dual echo data $(0.86 \times 0.86$ in-plane resolution; $2.5 \mathrm{~mm}$ slice thickness; TR 4,000 ms; TE1 $6.5 \mathrm{~ms}$; TE2 $102 \mathrm{~ms}$ ).

\section{fMRI protocol}

The functional dataset involves three cognitive tasks and two emotion tasks that assess cognitive thinking and emotion processes. The methods have been fully described 
Table 2 Cross-site validation of the T1 SPGR anatomical protocol

\begin{tabular}{|c|c|c|c|c|}
\hline Intra-class correlation & Stanford scan 1 & Stanford scan 2 & Sydney scan 1 & Sydney scan 2 \\
\hline Stanford scan 1 & 1.0 & & & \\
\hline Stanford scan 2 & 0.999 & 1.0 & & \\
\hline Sydney scan 1 & 0.996 & 0.996 & 1.0 & \\
\hline Sydney scan 2 & 0.997 & 0.996 & 0.996 & 1.0 \\
\hline Mean gray matter volume $\left(\mathrm{mm}^{3}\right)$ & $7,175 \pm 5,527$ & $7,198 \pm 5,552$ & $7,193 \pm 5,620$ & $7,226 \pm 5,668$ \\
\hline
\end{tabular}

Intra-class reliability coefficients for volumetric data estimated for 68 brain gray matter regions using the T1 SPGR MRI protocol, using repeat scans done at the Stanford and Sydney site magnetic resonance scanners. Data show that inter-site scan reliability (for example, Stanford scan 1 - Sydney scan 1 ) is high and on the same comparative level as within-site scans (for example, Sydney scan 1 and Sydney scan 2). The mean (+ SD) for gray matter volume across the 68 brain regions for each scan is also listed.

MRI Magnetic resonance imaging, SD Standard deviation, SPGR Spoiled gradient recalled.

elsewhere but are summarized below for completeness [21,22].

The cognitive tasks include an auditory oddball task, a continuous performance task and the Go-NoGo task. The oddball task is used for evaluating attention modulation. The stimuli consist of 20 high-pitched $(1,000 \mathrm{~Hz}$ at $75 \mathrm{~dB}$ ) 'target' tones and 100 lower-pitched $(500 \mathrm{~Hz}$ at $75 \mathrm{~dB}$ ) 'non-target' tones. In the continuous performance task, participants view a series of 120 letters (B, C, D or G) and are instructed to press a button when the same yellow letter appears twice in a row (a '1-back' design). To provide a baseline, 40 stimuli are presented in white, which participants are instructed to ignore. The Go-NoGo task is used to assess impulsivity (automatically-generated 'Go' responses) versus inhibition ('NoGo' responses). 'Go' responses are defined by the word 'press' presented in GREEN, while 'NoGo' responses are defined by the word 'press' presented in RED.

The emotion tasks consist of an unmasked conscious emotion processing task and a masked non-conscious emotion processing task. The unmasked emotion processing task $[23,24]$ was developed from a standardized set of 3D evoked facial expressions that depict fear, anger, disgust, sadness, happiness or are neutral [25]. There are a total of 240 stimuli grouped in blocks of eight faces of the same emotion, with each emotion block repeated five times. In the masked non-conscious emotion processing task, the same set of facial emotion stimuli are used but each stimulus consists of the emotion face presented nonconsciously (for $10 \mathrm{~ms}$ ), followed immediately by a neutral face mask stimulus (for $150 \mathrm{~ms}$ ). This paired presentation is designed to prevent conscious awareness of the emotion stimulus $[23,24]$.

\section{Data processing pipeline}

Data are processed using a custom-built automated pipeline run via scripts in the MATLAB 7.5 software (MathWorks, Natick, MA, USA). Processing tasks are performed by a cluster of 18 custom-built computer nodes running Linux (CENTOS 5.0 distribution); tasks are assigned, queued and coordinated using a central computer. Specific details of the analysis procedures are provided below. In summary, the raw DICOM data from the magnetic resonance scanner are imported using a script and converted to NIfTI format, then is run through sequential routines drawn from the Functional MRI of the Brain (FMRIB) Software Library (FSL) release 4.1.3 (http://www.fmrib.ox.ac.uk/fsl) [26], the Freesurfer image analysis suite (version 4.3) (documented and freely available for download online (http://surfer.nmr.mgh.harvard. edu/) [27], SPM8 software [28] and custom-written routines that have been described in previous publications [29-32]. Anonymized data are transferred between the two imaging sites via a secure ftp connection.

\section{Data storage}

Anonymized data are kept in a structured file system that includes selected intermediate steps to permit the easy recalculation of data for specific analyses. Off-site back-ups of data are kept in a secure facility.

\section{Specific MRI analyses}

\section{Volumetric and cortical thickness data}

Measurements of cortical thickness and volume are made as described previously [33] using Freesurfer. Briefly, cortical surface reconstruction and volumetric segmentation are performed in an automated manner on the 3D T1 weighted structural images using the Freesurfer image analysis suite (version 4.3). The full technical details of these procedures are described elsewhere [34-45]. All cortical segmentation and labels are manually inspected for accuracy. Voxel-based morphometry (VBM8) analysis as implemented in SPM8 is also performed.

\section{DTI processing - tract-based spatial statistical (TBSS) and tractography analysis}

We have previously presented the TBSS-based analysis used to process our DTI data [46]. DTI data are preprocessed and analyzed using the Oxford Centre for FMRIB Diffusion Toolbox and TBSS software tools, part of the FSL release 4.1.3 (http://www.fmrib.ox.ac.uk/fsl) $[26,47,48]$. The raw DTI data for each participant are 
Table 3 Detailed summary of the MRI acquisition parameters

\begin{tabular}{|c|c|c|c|}
\hline Domain & Task/Scan type & MRI/Autonomic measures & Scan protocol/Task description \\
\hline \multirow{6}{*}{$\begin{array}{l}\text { Structural } \\
\text { scans }\end{array}$} & \multirow[t]{3}{*}{$\mathrm{T} 1$} & Gray/white matter volume & \multirow{3}{*}{$\begin{array}{l}\text { Scan protocol: } 3 \mathrm{D} \text { T1 weighted high resolution SPGR MRI } \\
\text { scan, TR }=8.3 \mathrm{~ms}, \mathrm{TE}=3.2 \mathrm{~ms} \text {, Flip angle }=11^{0}, \mathrm{TI}=500 \mathrm{~ms} \text {, } \\
\mathrm{NEX}=1, \text { Matrix }=256 \times 256 \text {, resolution }=1 \mathrm{~mm} \times 1 \mathrm{~mm}, 180 \\
\text { contiguous } 1 \mathrm{~mm} \text { sagittal slices. }\end{array}$} \\
\hline & & Cortical thickness & \\
\hline & & Cortical surface area & \\
\hline & $\begin{array}{l}\text { Diffusion Tensor } \\
\text { Imaging (DTI) }\end{array}$ & $\begin{array}{l}\text { Fractional Anisotropy }(F A) \text {, mean }(M D) \text {, axial } \\
(A D) \text {, radial }(R D) \text { diffusivity measures for white } \\
\text { matter regions and fiber tracts }\end{array}$ & $\begin{array}{l}\text { Scan protocol: Spin echo DTI echo planar imaging scan, } 42 \\
\text { directions, } b \text { value }=1,250, \mathrm{TR}=17,000 \mathrm{~ms}, \mathrm{TE}=95 \mathrm{~ms}, \mathrm{NEX}=1 \text {, } \\
\text { Matrix }=128 \times 128 \text {, resolution }=1.72 \mathrm{~mm} \times 1.72 \mathrm{~mm}, 70 \\
\text { contiguous } 2.5 \mathrm{~mm} \text { axial/oblique slices covering the whole brain. }\end{array}$ \\
\hline & \multirow[t]{2}{*}{$\mathrm{PD} / \mathrm{T} 2$} & Proton density & \multirow{2}{*}{$\begin{array}{l}\text { Scan protocol: 2D Fast Spin echo scan, TR }=4,000 \mathrm{~ms} \text {, TE1 }= \\
6.4 \mathrm{~ms}, \mathrm{TE} 2=102 \mathrm{~ms}, \mathrm{NEX}=1, \text { Matrix }=256 \times 256 \text {, resolution }= \\
0.86 \mathrm{~mm} \times 0.86 \mathrm{~mm}, \mathrm{ETL}=16,70 \text { contiguous } 2.5 \mathrm{~mm} \text { axial/ } \\
\text { oblique slices covering whole brain. }\end{array}$} \\
\hline & & T2 structural scan for clinical evaluation & \\
\hline \multirow{4}{*}{$\begin{array}{l}\text { Functional } \\
\text { scans }\end{array}$} & \multirow[t]{3}{*}{ Oddball } & Changes in fMRI BOLD signal & \multirow{2}{*}{$\begin{array}{l}\text { Scan protocol: Echo planar imaging scan, TR }=2,500 \mathrm{~ms}, \mathrm{TE}= \\
27.5 \mathrm{~ms} \text {, Flip angle }=90^{\circ}, \mathrm{NEX}=1, \text { Matrix }=64 \times 64 \text {, resolution }= \\
3.75 \mathrm{~mm} \times 3.75 \mathrm{~mm}, 40 \text { contiguous } 3.5 \mathrm{~mm} \text { axial/oblique } \\
\text { slices covering whole brain in each volume, total } 120 \text { volumes. }\end{array}$} \\
\hline & & Average heart rate & \\
\hline & & Average skin conductance & $\begin{array}{l}\text { Task description: } 20 \text { target }(1,000 \mathrm{~Hz}) \text { and } 100 \text { nontarget } \\
(50 \mathrm{~Hz}) \text { tones presented one at a time at } 75 \mathrm{db}(50 \mathrm{~ms} \text { each, } \\
\mid \mathrm{SI}=2.4 \text { seconds). }\end{array}$ \\
\hline & Continuous performance & Changes in fMRI BOLD signal & Scan protocol: See Oddball protocol \\
\hline
\end{tabular}

$\begin{array}{ll}\text { test }(\mathrm{CPT}) & \text { Average heart rate } \\ & \text { Average skin conductance }\end{array}$

$\begin{array}{ll}\text { Go-NoGo } & \text { Changes in } \mathrm{fMRI} B O L D \text { signal } \\ & \text { Average heart rate } \\ & \text { Average skin conductance }\end{array}$

Emotion (masked 'nonconscious'; Then unmasked 'conscious')
Task description: 120 stimuli are presented (B, C, D or G letters, for $200 \mathrm{~ms}$ each, $\mid \mathrm{SI}=2.3 \mathrm{sec}$ ). Sixty were yellow letters to be held in working memory (no consecutive repetitions), 20 were 1-back sustained attention stimuli (consecutive repetitions of a letter in yellow) and 40 were perceptual baseline stimuli in white letters. Participants were measured for the number of errors or omissions.

\section{Scan protocol: See Oddball protocol}

Task description: 180 Go stimuli (word 'press' in green) and NoGo stimuli (word 'press' in red) each presented sequentially (500 ms each, ISI = .75 seconds). NoGo stimuli were not repeated more than three times in a row. Participants were measured for target detection rate, response time, errors of commission and errors of omission.

Scan protocol: See Oddball protocol

Task description: 240 3D evoked standardized facial expressions that depicted fear, anger, disgust, sadness, happiness or neutral were presented for $500 \mathrm{~ms}$ (ISI = .75 seconds). Stimuli were grouped into eight faces expressing the same emotion and were repeated five times in a pseudorandom order. Participants were assessed for explicit emotion processing for the 'Masked' condition.

Average skin conductance

The same set of facial expressions was presented for the 'Unmasked' condition; however, backward masking (expression presented for $10 \mathrm{~ms}$ immediately followed by a neutral face for $190 \mathrm{~ms}$ ) was used to prevent conscious awareness of facial expression.

Scan protocol: Dual echo MRI scan, TE1 $=10 \mathrm{~ms}, \mathrm{TE} 2=12.5 \mathrm{~ms}$, $\mathrm{TR}=700 \mathrm{~ms}$, Flip angle $=15^{\circ}, \mathrm{NEX}=1$, Matrix $=128 \times 128$, 40 contiguous $3.5 \mathrm{~mm}$ axial/oblique slices.

\author{
Additional Phase map \\ scan \\ Map magnetic field in homogeneities \\ for EPI distortion correction
}

2D Two-Dimensional, 3D Three-Dimensional, BOLD Blood oxygen level dependent; EPI, Echo-planar imaging, ETL Echo-train length, fMRI Functional magnetic resonance imaging, ISI Inter-stimulus interval, MRI Magnetic resonance imaging, NEX Number of excitations, PD Proton density, SPGR Spoiled gradient recalled, TE Echo time, TR Repetition time.

first corrected for head movement and eddy current distortions. Diffusion tensor models are then fitted independently for each voxel within the brain mask and images of fractional anisotropy (FA), mean diffusivity $(\mathrm{MD})$ and first $(\lambda 1)$, second $(\lambda 2)$ and third Eigen value $(\lambda 3)$ are generated for each participant. FA images from each participant are then aligned to the FMRIB58_FA template and transformed into Montreal Neurological Institute (MNI) $1521 \mathrm{~mm}^{3}$ standard space using FMRIB's nonlinear registration tool FNIRT $[49,50]$. Next, 
an average FA image is generated and thinned to create a white matter skeleton representing the centers of all white matter tracts common to all participants. This FA skeleton is then thresholded to FA $\geq 0.3$ to include the major white matter pathways but avoid peripheral tracts that are more vulnerable to inter-participant variability and/or partial volume effects with gray matter. Each participant's aligned FA image is then projected onto the mean FA skeleton. These skeletonized participant data will be used for performing voxel-wise cross-participant statistics using permutation testing procedures. Clusters with significant differences will be labeled using the Johns Hopkins University International Consortium for Brain Mapping (JHU ICBM)-DTI-81 white matter labels atlas [51]. DTI tractography will also be performed using a multi-fiber diffusion probabilistic model [52]. This method estimates probability distributions for one or more fiber populations at each brain voxel. DTI tractography will allow obtaining a more precise quantification of the exact orientation and trajectory of WM fiber tracts.

\section{Functional MRI data analysis}

The fMRI analysis procedures are described in detail elsewhere [22]. In brief, pre-processing and analysis are performed using SPM8 software (www.fil.ion.ucl.ac.uk/ spm), and involve motion correction, global signal estimation and removal, estimation of normalization transforms from functional to standard MNI space, spatial smoothing, and then application of a high-pass filter. A canonical hemodynamic response function (HRF)-convolved event-related model is used to model the blood oxygen level dependent (BOLD) responses for the three cognitive tasks. An HRF-convolved box-car function is used to model the BOLD response for both of the emotional processing tasks. Contrast images for each cognitive thinking and emotion processing task are generated and will be evaluated for voxel-wise comparisons in BOLD signal for the whole brain. A significance threshold of $P<0.05$ corrected for multiple comparisons will be used. Clusters with significant differences will be labeled using the Talairach Atlas.

\section{Region of interest definitions}

To test specific hypotheses of regional involvement in volume measurements, fMRI activation tasks and, in the resting state-fMRI, standardized regions of interest (ROI) have been defined using the WFU PickAtlas software as a basis [53]. For selected ROIs without standardized available masks, custom definitions will be generated using the automated coordinate extraction method within the NeuroSynth framework (http://neurosynth.org) [54]. This environment uses data derived from a machine-learning technique (naive Bayes classification) to estimate meta- analysis maps of activation maps based on specified search terms. For DTI data, the JHU white matter atlas will be used with the TBSS processed data to define white matter fiber bundles. DTI tractography will also be performed to define individualized fiber tracts for each participant.

\section{Quality control}

MRI data are evaluated at the time of acquisition by the radiographer. Each MRI study (excluding the fMRI data) is reported by a radiologist at the site of acquisition (Departments of Radiology, Westmead Hospital and Stanford University) to exclude structural abnormalities. The imported NIfTI files are visually inspected by the local imaging scientist prior to analysis. MRI protocols were standardized by using careful synchronization of parameters between sites, scanning of a human phantom on multiple occasions (MSK, SMG), inter-site visits and ongoing clinical assessment (see Table 2). Routine quality control of the analysis pipeline was performed using standardized outputs generated to reflect the quality of motion correction, image registration and parameter maps for the functional data, fractional anisotropy images for the DTI data and cortical-subcortical parcellations from Freesurfer for the structural T1 data.

\section{Analytic approach}

Analyses of iSPOT-D imaging data will be conducted in three phases.

\section{Phase 1 - identification of putative imaging biomarkers for MDD and subtype classification}

Phase 1 includes the first $15 \%$ of the MDD cohort tested at the Sydney site (that is, 30 MDD participants, 30 matched controls). Analyses will test for the convergence of structural and functional imaging data for brain circuitry dysfunction in depression (that is, Question 1). These analyses will replicate existing analyses in the literature using structural and functional imaging in MDD. We provide outcomes from these Phase 1 analyses in this report.

\section{Phase 2 - testing of biomarkers, hypothesis generation for testing against Phase 3 data}

Phase 2 includes the first $50 \%$ of the MDD cohort tested at the Sydney site (that is, 102 MDD participants with 34 in each of the three treatment arms, and 34 matched controls). Analyses will mainly identify baseline brain circuitry predictors of treatment response, core brain circuitry associated with depression and its subtypes, and brain circuits that change with treatment (that is, Questions 1 through 4).

\section{Phase 3 - replication of early findings, testing associations in pooled data}

Phase 3 includes the full MDD cohort tested at the Sydney site (204 MDD participants, 68 matched controls) 
and Stanford site (51 MDD participants, 17 matched controls). Analyses will replicate findings from Phase 2 analyses using the entire Sydney cohort and, a separate replication will be performed in an independent Stanford cohort. The issue of inter-site variance between the two imaging sites will be addressed prior to pooling data. We will first check for systematic differences, if these exist then they will be modeled out from the analysis, using site as a covariate. The full cohort will also provide sufficient power to test for associations of imaging measures with other data available from the iSPOT-D study protocol (Question 5).

As a first pass, each of the MRI measures will be analyzed independently and then combined into a multimodal multiple regression analysis.

\section{Power Calculation/Effect size}

For analysis of predictive measures (for example, imaging measures that predict treatment response or remission due to a specific agent, or predict treatment resistance assuming that about $50 \%$ of participants will respond [55]) at a statistical power of $80 \%$ and an effect size of 1 standard deviation, the sample size required is 17 , (that is, assuming each treatment has $\mathrm{N}$ of approximately $102 / 3->\mathrm{N}=34$; meaning $\mathrm{n}(\mathrm{R})=34^{*} .5=17$ responders/non-responders). This permits us to analyze single treatment arms at the 50\% data level, enabling replication of effects with the second $50 \%$ of data.

\section{Future exploratory analyses}

The high-powered imaging dataset available from the iSPOT-D study will also provide the opportunity for new analyses on the cutting edge of the imaging field. Below, we list some of the novel analyses that we plan to conduct using the imaging data.

\section{Whole brain mapping of inter-regional connectivity using DTI}

We quantify inter-regional white matter connectivity by defining cortical ROIs based on the cortical thickness parcellation, then determining the number of fibers connecting each region. Fiber tracking utilizes a multifiber diffusion probabilistic model that estimates probability distributions for one or more fiber populations at each brain voxel [52]. Tractography is performed by sequentially using each of the cortical labels as seed and the remaining labels as targets. The resulting dataset, a $70 \times$ 70 inter-regional connectivity matrix for each participant, will then be subjected to either a pattern-based analysis (for example, principle components analysis [56] or group theory [57-60]), or a focused, hypothesis-driven analysis of specific inter-regional connectivity. This method has been previously described using Phase 1 data from the first 15\% of the iSPOT-D dataset [56]. We used a pattern analysis approach to analyze these data to show a $92 \%$ accuracy in characterizing the MDD and control cohorts.

\section{White matter hyperintensity (WMH) analysis}

WMH will be measured using a custom, semi-automated routine using dual-echo data (proton density and spin echo data) combined with reformatted T1W data in the axial plane. Initial lesions are selected using OsiriX (http:// www.osirix-viewer.com) [61]; then lesions are quantified in MATLAB using a multi-modality (T1, T2, Proton Density) cluster analysis technique (details to be published in a future paper). A separate qualitative analysis will also be performed using the well-validated Schelton's score [62].

\section{Volume and connectivity analysis of the basal ganglia, amygdala, thalamus, brainstem and cerebellum}

Several novel analytical approaches currently underway are designed to obtain more detailed connectivity information from the deep gray structures and from the cerebellum and brainstem structures, along the lines of previous probabilistic approaches [47].

\section{Resting state fMRI analysis}

The fMRI task data will be used to extract resting state fMRI data. To identify resting state connectivity, the task effects are modeled within a general linear model framework. These effects are removed and the remaining (residual) time-series signal is analyzed using SPM8 software. This method of obtaining resting connectivity from task fMRI data has been previously established and validated against non-task-derived resting state data $[63,64]$.

\section{Small world analysis of brain networks}

The application of graph theory methods to imaging data has been useful in extracting sensitive measures of brain connectivity [57-60]. We will test how these measures are altered in MDD and test their prognostic value.

\section{Results}

Here we present a summary of outcomes to date from the analysis of Phase 1 using multimodality data (DTI, fMRI and T1). Using these measures, we test for convergent evidence of core brain circuitry dysfunction in depression, looking at the baseline data for the first $15 \%$ of subjects (prior to treatment). For this analysis, we investigate the mechanisms of this circuitry, with a specific focus on the dorsolateral prefrontal cortex, anterior cingulate cortex (ACC) and amygdala.

Our preliminary data (first $15 \%$ of subjects) demonstrate prefrontal dysfunction in MDD, with contributions from DTI, fMRI and T1 datasets. In the melancholic MDD subgroup, we have shown alterations of white matter within the white matter fiber tracts that are associated with the prefrontal, limbic and thalamic circuitry 
$[46,56]$. For the same cohort, we also found prefrontal dysfunction using fMRI data from all five cognitive and emotion processing tasks: MDD participants were distinguished by a distinctive biosignature of hypoactivation of the dorsolateral prefrontal cortex during working memory updating and during conscious negative emotion processing, hyperactivation of the dorsomedial prefrontal cortex (ACC) during working memory and response inhibition cognitive tasks, and hypoactivation of the dorsomedial prefrontal cortex (ACC) during conscious processing of positive emotion tasks [22].

Further data from the first $15 \%$ of subjects highlight the central role of the limbic regions and the ACC in MDD using convergent evidence from fMRI, structural T1 and DTI data. The fMRI data and DTI data were analyzed using the methods described in Korgaonkar et al.

\section{a Control $\square$ MDD}
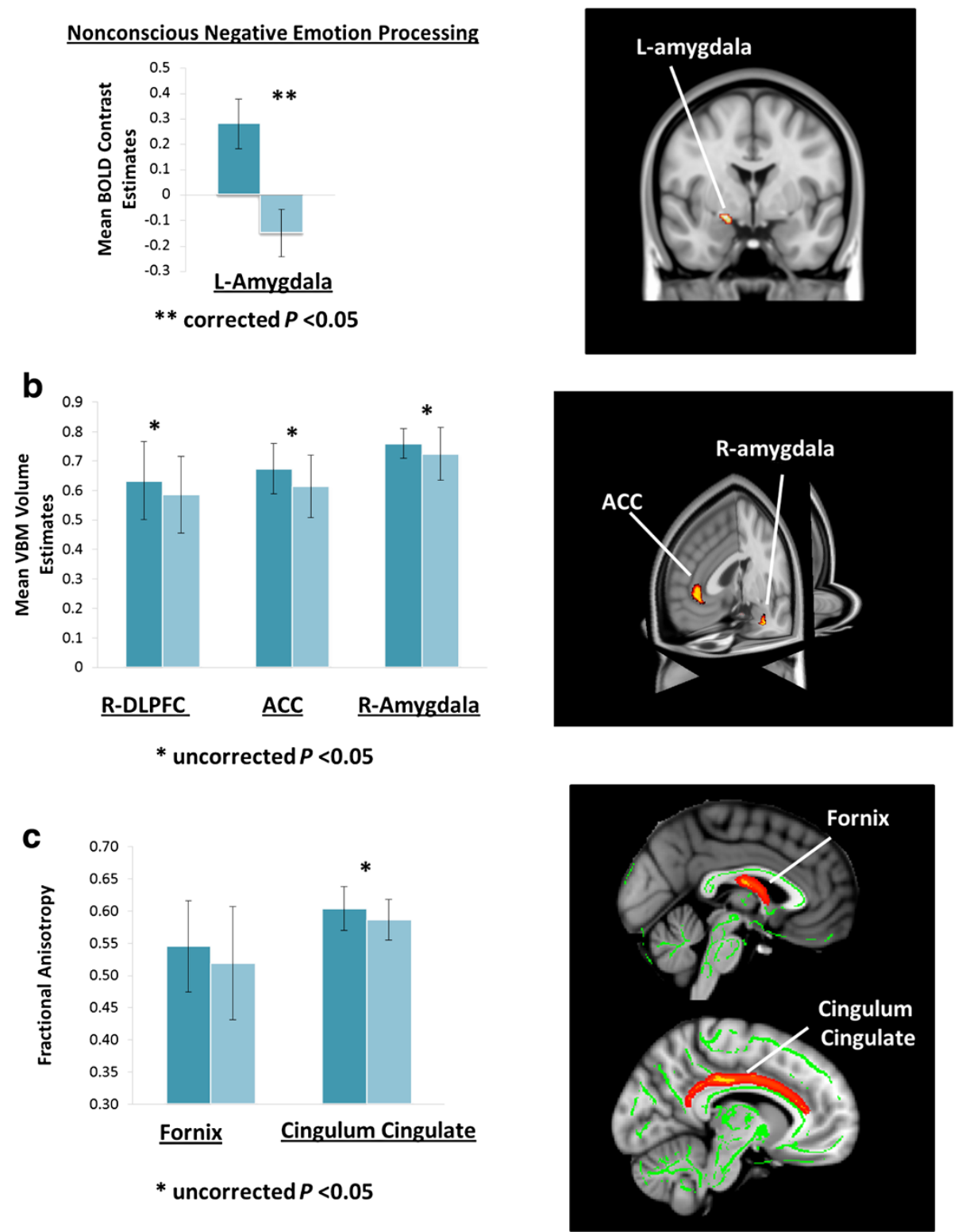

Figure 2 Summary of preliminary functional MRI data from the first $15 \%$ of participants (MDD versus controls). (a) A coronal view (right) demonstrating significant hypoactivation of the left amygdala for non-conscious negative emotion processing. Comparison between the MDD and control groups (left) shows a significant difference (corrected $P<0.05$ ). (b) Significant reductions in gray matter volume (uncorrected $P<0.05$ ) are shown in the right amygdala, right dorsolateral prefrontal cortex and anterior cingulate regions. (c) FA in the fornix and cingulum portion of the cingulate bundle was significantly lower $(P<0.05)$ in the MDD group in comparison to controls. 
$[22,46]$, while the structural $\mathrm{T} 1$ weighted data were analyzed using the methods described above. We tested for MDD-Control group differences in the FMRI BOLD signal and gray matter volume in the ACC and amygdala, as well as for FA differences in two white matter fiber bundles associated with these regions, that is, the cingulum cingulate bundle and fornix respectively. A significant difference (corrected $P<0.05$ ) between the MDD and control group was found in the left amygdala for non-conscious negative emotion processing, with hypoactivation of this region seen in the MDD group (Figure 2a) (significant differences in ACC and DLPFC are already reported in [22]). A reduction in gray matter volume (uncorrected $P<0.05$ ) was also seen in both the amygdala and ACC regions (Figure $2 \mathrm{~b}$ ). FA in the cingulum cingulate bundle was also found to be significantly reduced $(P<0.05)$ in the MDD group in comparison to controls (Figure 2c).

\section{Discussion}

There are currently no clinically useful predictors of MDD response or remission with the antidepressants escitalopram, sertraline or venlafaxine-XR. MRI measures of brain circuitry and function hold great potential as "biomarkers" in psychiatric disease; however, there is a lack of data regarding their use in predicting treatment response in MDD. The imaging sub-study of iSPOT-D seeks to provide high-level evidence regarding which aspects of brain circuitry predict and moderate response to these antidepressants.

In this paper, we have outlined the protocol and rationale for the imaging sub-study of iSPOT-D. We summarized the already-published outcomes from the first 15\% of the data and also presented some additional supportive data. These multimodality outcomes provide the first convergent evidence of dysregulation in both the activation and connectivity of the prefrontal-limbic circuitry in depression. Analysis of the $50 \%$ data will focus on predictive measures of treatment response, with an a priori focus on these circuits. The careful design of our study, its power and the focus on multimodality data maximize the possibility of achieving our aim of generating imaging-based metrics that have clinically relevant predictive value.

At the completion of iSPOT-D and the imaging substudy delineated here, we will investigate how imaging measures of brain circuitry relate to other clinical, genetic, behavioral and physiological units of analysis in order to test combined predictors of treatment outcome.

\section{Trial status}

Enrollment is ongoing. Final data collection date for primary outcome measure is estimated to be August 2013. Study completion date is estimated to be December 2013.

\section{Abbreviations}

2D: Two-dimensional; 3D: Three-dimensional; ACC: Interior cingulate cortex; ADM: Antidepressant medication; BOLD: Blood oxygen level dependent; DSM-5: the fifth edition of the Diagnostic and Statistical Manual of Mental Disorders; DTI: Diffusion tensor imaging; EPI: Echo-planar imaging; ETL: Echotrain length; FA: Fractional anisotropy; fMRI: Functional magnetic resonance imaging; FMRIB: Functional MRI of the brain; FSL: Functional MRI of the brain software library; HRF: Hemodynamic response function; $\mathrm{HRSD}_{17}$ : 17-item Hamilton Rating Scale for Depression; ISI: Inter-stimulus interval; iSPOTD: International study to protect optimized treatment for depression; JHU ICBM: Johns Hopkins University International Consortium for Brain Mapping; MD: Mean diffusivity; MDD: Major depressive disorder; MINI: Mini-

international neuropsychiatric interview; MNI: Montreal neurological institute; MRI: Magnetic resonance imaging; NEX: Number of excitations; PET: Positron emission tomography; ROI: Regions of interest; SD: Standard deviation; SNRIs: Selective norepinephrine reuptake inhibitors; SPGR: Spoiled gradient recalled; SSRIs: Selective serotonin reuptake inhibitors; TBSS: Tract-based spatial statistical; TE: Echo time; TR: Repetition time; VBM8: Voxel-based morphometry; WMH: White matter hyperintensity; $\lambda 1$ : First Eigen value; $\lambda 2$ : Second Eigen value; $\lambda 3$ : Third Eigen value.

\section{Competing interests}

SMG has received consulting fees from the Brain Resource Company. AH has received consultancy fees from Eli Lilly, Janssen-Cilag and Lundbeck Australia. He has received payments for educational sessions run for Astra Zeneca, Janssen Cilag and Eli Lilly. He has run educational sessions Reed Business Information. He is an investigator on industry sponsored trials by Hoffman-La Roche, Janssen-Cilag Australia and Brain Resource Company. SHK serves as a consultant and has stock options with Brain Resource Company. SW has received fees as a consultant from Cyberoniclnc, ImaRx Therapeutics Inc, Bristol-Myers Squibb Company, Organon, Case-Western University and Singapore Clinical Research Institute. AFS has received fees as a consultant from Brain Cells, CeNeRxm, CNS Response, Corcept, Glaxo-Smith Kline, Merck and Company, Neuronectics, PharmaNeuroBoost, Sanofi-Aventis and Takeda. He is a stock holder in Amnestix, Brain Cells, CeNeRx, Corpect, Forest, Merck and Company, Neurocrine, Pfizer, PharmaNeuroBoost, Somaxon and Synosis. CBN has received a Scientific Advisory Board/Board of Directors fee from AstraZeneca, PharmaNeuroboost, Forest Laboratories, NARSAD, Quintiles, Janssen/Ortho-McNeil, Mt. Cook Pharmalnc, George West Mental Health Foundation and NovaDelPharma. He holds stock in Corcept, CeNeRx, ReVax, PharmaNeuroboost and NovadelPharma. CBN has patents for methods and devices for the transdermal delivery of lithium (US 6,375,990 BI) and a method to estimate serotonin and norepinephrine transporter occupancy after drug treatment using patient or animal serum (provisional filing April, 2001). EG is founder of Brain Resource Ltd and receives income as its Chief Executive Officer and Chairman. He has stock options in Brain Resource Ltd. LMW has received consulting fees and stock options in Brain Resource Ltd, and is a stock holder in Brain Resource Ltd. She has received Advisory Board fees from Pfizer

MSK and AE have no competing interests to disclose.

\section{Authors' contributions}

SMG made substantial contributions to conception and design of the trial, provided important intellectual content to the manuscript, and was substantially involved in drafting and revising the manuscript. MSK, AE, AH, SHK, SW, AS, CBN and EG made substantial contributions to the conception of the trial, and were substantially involved in drafting and revising the manuscript. LMW made substantial contributions to the conception and design of the trial, provided important intellectual content to the manuscript, and was substantially involved in drafting and revising the manuscript. All authors gave final approval of the submitted version of the manuscript.

\section{Authors' information}

SMG is an Associate Professor at the Sydney Medical School in the University of Sydney. He designed the imaging protocol for the study and with MSK has led the analysis and processing of the imaging data. He is a clinicianscientist with a strong interest in translational imaging. He completed his specialty training in radiology at Royal Prince Alfred Hospital and his doctorate in MRI imaging of the brain at Oxford University. His clinical and research interests are focused on cardiac and neuroimaging. He is currently a 
Sydney Medical Foundation Fellow at the University of Sydney and Head of Imaging at the Brain Dynamics Center.

MSK is a Senior Imaging Scientist on the study. He completed his Masters in Medical Physics and doctoral degree in MR Imaging at the department of Biomedical Engineering at Stony Brook University, NY. He is currently appointed as Senior Imaging Scientist and Director of Brain Dynamics Center, Sydney Medical School - Westmead.

AE is Assistant Professor of Psychiatry and Behavioral Sciences at Stanford University, an Investigator in the Sierra-Pacific Mental Illness Research Education and Clinical Center (MIRECC) at the Veterans Affairs Palo Alto Health Care System, and a board-certified psychiatrist. He directs a laboratory focused on understanding the neurobiology of mood and anxiety disorders. $\mathrm{AH}$ is an Associate Professor in the Discipline of Psychiatry at the University of Sydney and is the Clinical Director at the Brain Dynamics Centre, Westmead Millennium Institute at the University of Sydney. He is a senior staff specialist for the Western Sydney Local Health Network. His interests have centered upon the treatment, psychophysiology and neuroimaging of young people with psychosis and depression.

SHK is a senior research fellow in the Miami University School of Psychiatry and Behavioral Sciences and a Director on the board of the BRAINnet Foundation. He recently served as the Research Director for the American Foundation for Suicide Prevention. He was the first Director of the Neuroscience Research Branch at the NIMH where he was responsible for initiating new research programs, including Human Brain Imaging and the Human Brain Project. SHK was founder of the International Neuroinformatics Coordinating Facility. He currently serves as a consultant to Brain Resources. SW is a professor in the Department of Epidemiology and Co-Director of the Epidemiology Data Center, University of Pittsburgh. His secondary appointment is in psychiatry. He is an Associate Dean for Research in the Graduate School of Public Health.

AFS is the Kenneth T. Norris, Jr. Professor in Psychiatry and Behavioral Sciences at Stanford University. Before this, he built the department of Psychiatry and Behavioral Sciences as chair of the department.

CBN is the Leonard M. Miller Professor and Chairman of the Department of Psychiatry and Behavioral Sciences, University of Miami.

EG is the founder and Chairman of Brain Resource, which has established the first standardized battery for assessing human behavior and brain function and structure.

LMW is acting Professor in Psychiatry and Behavioral Sciences at Stanford University, and holds a joint appointment as Professor in Cognitive Neuropsychiatry at Sydney Medical School.

\section{Acknowledgements}

We acknowledge Brain Resource as the sponsor for the iSPOT-D study (NCT00693849). Claire Day (Global Study Co-ordinator) is thanked for her pivotal role in making this study happen, and for her boundless enthusiasm and energy. We thank the iSPOT-D Executive Committee for their valuable input into this manuscript and into the study overall (Members: A John Rush (Chair), Lea Williams (Academic PI), Steve Koslow, AmitEtkin, Evian Gordon, Steve Koslow, Stephen R Wisniewski. We also thank those responsible for designing the iSPOT-D protocol (A. John Rush, Lea M Williams, Evian Gordon, Charles B Nemeroff, Alan F Schatzberg). We gratefully acknowledge the editorial support of Jon Kilner, MS, MA (Pittsburgh, PA, USA). SMG acknowledges the support of the Sydney University Medical Foundation. Dr Lavier Gomes, Ms Sheryl Foster and the Department of Radiology at Westmead are thanked for their substantial contributions to MRI data acquisition.

\section{Author details}

${ }^{1}$ The Brain Dynamics Centre, University of Sydney Medical School Westmead and Westmead Millennium Institute, Sydney, NSW 2145, Australia. ${ }^{2}$ Brain Resource, Level 12, 235 Jones Street, Ultimo, Sydney, NSW 2007, Australia and Suite 200, 1000 Sansome Street, San Francisco, CA 94111, USA. ${ }^{3}$ Sydney Medical School, University of Sydney, Camperdown, NSW 2050, Australia. ${ }^{4}$ Department of Radiology, Royal Prince Alfred Hospital, Camperdown, NSW 2050, Australia. ${ }^{5}$ Discipline of Psychiatry, University of Sydney Medical School: Western, Westmead Hospital, Sydney, NSW 2145, Australia. ${ }^{6}$ Department of Psychiatry and Behavioral Sciences, Stanford University, 401 Quarry Road, Stanford, CA 94305, USA. ${ }^{7}$ Sierra-Pacific Mental IIIness Research, Education, and Clinical Center (MIRECC), Veterans Affairs Palo Alto Health Care System, 3801 Miranda Ave, Palo Alto, CA 94304, USA. ${ }^{8}$ Department of Psychiatry and Behavioral Sciences, University of Miami Miller
School of Medicine, Miami, FL 33136, USA. ${ }^{9}$ BRAINnet Foundation, 71 Stephenson Street, Suite 400, San Francisco, CA 94105, USA.

Received: 4 February 2013 Accepted: 4 July 2013

Published: 18 July 2013

\section{References}

1. Mathers C, Fat DM, Boerma JT: The Global Burden of Disease: 2004 update Geneva: World Health Organization; 2008.

2. Exner C, Lange C, Irle E: Impaired implicit learning and reduced presupplementary motor cortex size in early-onset major depression with melancholic features. J Affect Disord 2009, 119:156-162.

3. Shah PJ, Glabus MF, Goodwin GM, Ebmeier KP: Chronic, treatmentresistant depression and right fronto-striatal atrophy. Br J Psychiatry 2002, 180:434-440.

4. Soriano-Mas C, Hernández-Ribas R, Pujol J, Urretavizcaya M, Deus J, Harrison BJ, Ortiz H, López-Solà M, Menchón JM, Cardoner N: Cross-sectional and longitudinal assessment of structural brain alterations in melancholic depression. Biol Psychiatry 2011, 69:318-325.

5. Greenberg P, Corey-Lisle PK, Birnbaum H, Marynchenko M, Claxton A: Economic implications of treatment-resistant depression among employees. Pharmacoeconomics 2004, 22:363-373.

6. Williams LM, Rush AJ, Koslow SH, Wisniewski SR, Cooper NJ, Nemeroff CB, Schatzberg AF, Gordon E: International study to predict optimized treatment for depression (iSPOT-D), a randomized clinical trial: rationale and protocol. Trials 2011, 12:4.

7. Igelstrom KM, Heyward PM: Inhibition of hippocampal excitability by citalopram. Epilepsia 2012, 53:2034-2042.

8. Riedel WJ, Eikmans K, Heldens A, Schmitt JA: Specific serotonergic reuptake inhibition impairs vigilance performance acutely and after subchronic treatment. J Psychopharmacol 2005, 19:12-20.

9. Arnone D, McKie S, Elliott R, Thomas EJ, Downey D, Juhasz G, Williams SR, Deakin JF, Anderson IM: Increased amygdala responses to sad but not fearful faces in major depression: relation to mood state and pharmacological treatment. Am J Psychiatry 2012, 169:841-850.

10. Ruhe HG, Booij J, van Weert HC, Reitsma JB, Franssen EJ, Michel MC, Schene A: [Dose-escalation of SSRIS in major depressive disorder. Should not be recommended in current guidelines]. Tijdschr Psychiatr 2010, 52:615-625.

11. Victor TA, Furey ML, Fromm SJ, Bellgowan PS, Ohman A, Drevets WC: The extended functional neuroanatomy of emotional processing biases for masked faces in major depressive disorder. PLoS One 2012, 7:e46439.

12. Phan KL, Coccaro EF, Angstadt M, Kreger KJ, Mayberg HS, Liberzon I, Stein $M B$ : Corticolimbic brain reactivity to social signals of threat before and after sertraline treatment in generalized social phobia. Biol Psychiatry 2013, 73:329-336.

13. Charney D, Barlow D, Botteron K, Cohen J: Neuroscience Research Agenda to Guide Development of a Pathophysiologically Based Classification System. In A research agenda for DSM-V. Edited by Kupfer DJ, First MB, Regier DA. American Psychiatric Publishing, Incorporated; 2002:31-84.

14. National Institute of Mental Health: The National Institute of Mental Health Strategic Plan. Bethesda, MD: National Institute of Mental Health. NIH publication 08-6368; 2008.

15. Insel T, Cuthbert B, Garvey M: Research Domain Criteria (RDoC): toward a new classification framework for research on mental disorders. Am J Psychiatry 2010, 167:748-751.

16. Koslow SH, Williams LM, Gordon E: Personalized medicine for the brain: a call for action. Mol Psychiatry 2010, 15:229-230.

17. Atkinson AJ, Colburn WA, DeGruttola VG, DeMets DL, Downing GJ, Hoth DF, Oates JA, Peck CC, Schooley RT, Spilker BA, Woodcock J, Zeger SL: Biomarkers and surrogate endpoints: preferred definitions and conceptual framework. Clin Pharmacol Ther 2001, 69:89-95.

18. Rush AJ, Fava M, Wisniewski SR, Lavori PW, Trivedi MH, Sackeim H, Thase ME, Nierenberg A, Quitkin FM, Kashner TM, Kupfer DJ, Rosenbaum JF, Alpert J, Stewart JW, McGrath PJ, Biggs MM, Shores-Wilson K, Lebowitz BD, Ritz L, Niederehe G, STAR*D Investigators Group: Sequenced treatment alternatives to relieve depression (STAR*D): rationale and design. Control Clin Trials 2004, 25:119-142.

19. Australian Government: Department of Health and Ageing: Therapeutic Goods Administration. http://www.tga.gov.au/docs/about/publications.htm.

20. CFR - Code of Federal Regulations Title 21. http://www.accessdata.fda.gov/ scripts/cdrh/cfdocs/cfffr/cfrsearch.cfm?cfrpart = 312 . 
21. Gatt JM, Korgaonkar MS, Schofield PR, Harris A, Clark CR, Oakley KL, Ram K, Michaelson H, Yap S, Stanners M, Wise V, Williams LM: The TWIN-E project in emotional wellbeing: study protocol and preliminary heritability results across four MRI and DTI measures. Twin Res Hum Genet 2012, 15:419-441.

22. Korgaonkar MS, Grieve SM, Etkin A, Koslow SH, Williams LM: Using standardized $\mathrm{FMRI}$ protocols to identify patterns of prefrontal circuit dysregulation that are common and specific to cognitive and emotional tasks in major depressive disorder: first wave results from the iSPOT-D study. Neuropsychopharmacology 2013, 38:863-871.

23. Liddell BJ, Williams LM, Rathjen J, Shevrin H, Gordon E: A temporal dissociation of subliminal versus supraliminal fear perception: an eventrelated potential study. J Cogn Neurosci 2004, 16:479-486.

24. Williams LM, Palmer D, Liddell BJ, Song L, Gordon E: The 'when' and 'where' of perceiving signals of threat versus non-threat. Neuroimage 2006, 31:458-467.

25. Gur RC, Sara R, Hagendoorn M, Marom O, Hughett P, Macy L, Turner T, Bajcsy R, Posner A, Gur RE: A method for obtaining 3-dimensional facial expressions and its standardization for use in neurocognitive studies. J Neurosci Methods 2002, 115:137-143.

26. Smith SM, Jenkinson M, Woolrich MW, Beckmann CF, Behrens TEJ, Johansen-Berg H, Bannister PR, De Luca M, Drobnjak I, Flitney DE, Niazy RK, Saunders J, Vickers J, Zhang Y, De Stefano N, Brady JM, Matthews PM: Advances in functional and structural MR image analysis and implementation as FSL. Neuroimage 2004, 23(Suppl 1):S208-S219.

27. Fischl B: FreeSurfer. Neuroimage 2012, 62:774-781.

28. Frackowiak RSJ, Ashburner JT, Penny WD, Zeki S: Human Brain Function. 2nd edition. Waltham, MA: Academic; 2004.

29. Grieve SM, Clark CR, Williams LM, Peduto AJ, Gordon E: Preservation of limbic and paralimbic structures in aging. Hum Brain Mapp 2005, 25:391-401.

30. Grieve SM, Williams LM, Paul RH, Clark CR, Gordon E: Cognitive aging, executive function, and fractional anisotropy: a diffusion tensor MR imaging study. AJNR Am J Neuroradiol 2007, 28:226-235.

31. Paul R, Henry L, Grieve SM, Guilmette TJ, Niaura R, Bryant R, Bruce S, Williams LM, Richard CC, Cohen RA, Gordon E: The relationship between early life stress and microstructural integrity of the corpus callosum in a non-clinical population. Neuropsychiatr Dis Treat 2008, 4:193-201.

32. Seckfort D, Paul R, Grieve S, Vandenberg B, Bryant R, Williams L, Clark C, Cohen R, Bruce S, Gordon E: Early life stress on brain structure and function across the lifespan: a preliminary study. Brain Imaging Behav 2008, 2:49-58.

33. Grieve SM, Korgaonkar MS, Clark CR, Williams LM: Regional heterogeneity in limbic maturational changes: evidence from integrating cortical thickness, volumetric and diffusion tensor imaging measures. Neuroimage 2011, 55:868-879.

34. Fischl B, Sereno MI, Tootell RB, Dale AM: High-resolution intersubject averaging and a coordinate system for the cortical surface. Hum Brain Mapp 1999, 8:272-284.

35. Fischl B, Sereno MI, Dale AM: Cortical surface-based analysis. II: inflation, flattening, and a surface-based coordinate system. Neuroimage 1999, 9:195-207.

36. Dale AM, Fischl B, Sereno MI: Cortical surface-based analysis. I. Segmentation and surface reconstruction. Neuroimage 1999, 9:179-194.

37. Dale AM, Sereno MI: Improved localization of cortical activity by combining Eeg and Meg with Mri Cortical surface reconstruction - a linear-approach. J Cogn Neurosci 1993, 5:162-176.

38. Fischl B, Dale AM: Measuring the thickness of the human cerebral cortex from magnetic resonance images. Proc Natl Acad Sci U S A 2000, 97:11050-11055

39. Fischl B, Liu A, Dale AM: Automated manifold surgery: constructing geometrically accurate and topologically correct models of the human cerebral cortex. IEEE Trans Med Imaging 2001, 20:70-80.

40. Fischl B, Salat DH, Busa E, Albert M, Dieterich M, Haselgrove C, van der Kouwe A, Killiany R, Kennedy D, Klaveness S, Montillo A, Makris N, Rosen B, Dale AM: Whole brain segmentation: automated labeling of neuroanatomical structures in the human brain. Neuron 2002, 33:341-355.

41. Fischl B, Salat DH, van der Kouwe AJ, Makris N, Segonne F, Quinn BT, Dale AM: Sequence-independent segmentation of magnetic resonance images. Neuroimage 2004, 23(Suppl 1):S69-S84.

42. Han X, Jovicich J, Salat D, van der Kouwe A, Quinn B, Czanner S, Busa E, Pacheco J, Albert M, Killiany R, Maguire P, Rosas D, Makris N, Dale A,
Dickerson B, Fischl B: Reliability of MRI-derived measurements of human cerebral cortical thickness: the effects of field strength, scanner upgrade and manufacturer. Neuroimage 2006, 32:180-194.

43. Jovicich J, Czanner S, Greve D, Haley E, van der Kouwe A, Gollub R, Kennedy D, Schmitt F, Brown G, Macfall J, Fischl B, Dale A: Reliability in multi-site structural MRI studies: effects of gradient non-linearity correction on phantom and human data. Neuroimage 2006, 30:436-443.

44. Segonne F, Dale AM, Busa E, Glessner M, Salat D, Hahn HK, Fischl B: A hybrid approach to the skull stripping problem in MRI. Neuroimage 2004, 22:1060-1075.

45. Fischl B, van der Kouwe A, Destrieux C, Halgren E, Ségonne F, Salat DH, Busa E, Seidman LJ, Goldstein J, Kennedy D, Caviness V, Makris N, Rosen B, Dale AM: Automatically parcellating the human cerebral cortex. Cereb Cortex 2004, 14:11-22.

46. Korgaonkar MS, Grieve SM, Koslow SH, Gabrieli JD, Gordon E, Williams LM: Loss of white matter integrity in major depressive disorder: evidence using tract-based spatial statistical analysis of diffusion tensor imaging. Hum Brain Mapp 2011, 32:2161-2171.

47. Behrens TE, Johansen-Berg H, Woolrich MW, Smith SM, Wheeler-Kingshott CM, Boulby P, Barker GJ, Sillery EL, Sheehan K, Ciccarelli O, Thompson AJ, Brady JM, Matthews PM: Non-invasive mapping of connections between human thalamus and cortex using diffusion imaging. Nat Neurosci 2003, 6:750-757.

48. Smith SM, Jenkinson M, Johansen-Berg H, Rueckert D, Nichols TE, Mackay CE, Watkins KE, Ciccarelli O, Cader MZ, Matthews PM, Behrens TE: Tractbased spatial statistics: voxelwise analysis of multi-subject diffusion data. Neuroimage 2006, 31:1487-1505.

49. Andersson JL, Jenkinson M, Smith S: Non-linear Registration aka Spatial Normalisation. FMRIB Technical Report TR07JA2. 2007. www.fmrib.ox.ac.uk analysis/techrep.

50. Andersson $J$, Jenkinson M, Smith S: Non-linear Optimisation. FMRIB Technical Report TR07JA1. 2007 www.fmrib.ox.ac.uk/analysis/techrep.

51. Mori S, Oishi K, Jiang H, Jiang L, Li X, Akhter K, Hua K, Faria AV, Mahmood A, Woods R, Toga AW, Pike GB, Neto PR, Evans A, Zhang J, Huang H, Miller MI, van Zijl P, Mazziotta J: Stereotaxic white matter atlas based on diffusion tensor imaging in an ICBM template. Neuroimage 2008, 40:570-582.

52. Behrens TE, Berg HJ, Jbabdi S, Rushworth MF, Woolrich MW: Probabilistic diffusion tractography with multiple fibre orientations: what can we gain? Neuroimage 2007, 34:144-155.

53. Ja M, Laurienti PJ, Ra K, Burdette JH: An automated method for neuroanatomic and cytoarchitectonic atlas-based interrogation of fMRI data sets. Neuroimage 2003, 19:1233-1239.

54. Yarkoni T, Poldrack RA, Nichols TE, Van Essen DC, Wager TD: Large-scale automated synthesis of human functional neuroimaging data. Nat Methods 2011, 8:665-670.

55. Trivedi MH, Rush AJ, Wisniewski SR, Nierenberg AA, Warden D, Ritz L, Norquist G, Howland RH, Lebowitz B, McGrath PJ, Shores-Wilson K, Biggs MM, Balasubramani GK, Fava M, STAR*D Study Team: Evaluation of outcomes with citalopram for depression using measurement-based care in STAR* $D$ : implications for clinical practice. Am J Psychiatry 2006, 163:28-40.

56. Korgaonkar MS, Cooper NJ, Williams LM, Grieve SM: Mapping inter-regional connectivity of the entire cortex to characterize major depressive disorder: a whole-brain diffusion tensor imaging tractography study. Neuroreport 2012, 23:566-571.

57. He H, Sui J, Yu Q, Turner J, Ho B-C, Sponheim SR, Manoach DS, Clark VP, Calhoun VD: Altered small-world brain networks in schizophrenia patients during working memory performance. PLOS One 2012, 7:e38195.

58. Milo R, Shen-Orr S, Itzkovitz S, Kashtan N, Chklovskii D, Alon U: Network motifs: simple building blocks of complex networks. Science 2002, 298:824-827.

59. Wang J-H, Zuo X-N, Gohel S, Milham MP, Biswal BB, He Y: Graph theoretical analysis of functional brain networks: test-retest evaluation on shortand long-term resting-state functional MRI data. PLoS One 2011, 6:e21976.

60. Zhao X, Liu Y, Wang X, Liu B, Xi Q, Guo Q, Jiang H, Jiang T, Wang P. Disrupted small-world brain networks in moderate Alzheimer's disease: a resting-state FMRI study. PLoS One 2012, 7:e33540.

61. Rosset A, Spadola L, Ratib O: OsiriX: an open-source software for navigating in multidimensional DICOM images. J Digit Imaging 2004 $17: 205-216$

62. Scheltens P, Barkhof F, Leys D, Pruvo JP, Nauta JJ, Vermersch P, Steinling M Valk J: A semiquantative rating scale for the assessment of signal 
hyperintensities on magnetic resonance imaging. J Neurol Sci 1993, 114:7-12.

63. Fair DA, Schlaggar BL, Cohen AL, Miezin FM, Dosenbach NU, Wenger KK, Fox MD, Snyder AZ, Raichle ME, Petersen SE: A method for using blocked and event-related fMRI data to study "resting state" functional connectivity. Neuroimage 2007, 35:396-405.

64. Gavrilescu M, Stuart GW, Rossell S, Henshall K, McKay C, Sergejew AA, Copolov D, Egan GF: Functional connectivity estimation in fMRI data: influence of preprocessing and time course selection. Hum Brain Mapp 2008, 29:1040-1052.

doi:10.1186/1745-6215-14-224

Cite this article as: Grieve et al:: Brain imaging predictors and the

international study to predict optimized treatment for depression: study protocol for a randomized controlled trial. Trials 2013 14:224.

\section{Submit your next manuscript to BioMed Central and take full advantage of:}

- Convenient online submission

- Thorough peer review

- No space constraints or color figure charges

- Immediate publication on acceptance

- Inclusion in PubMed, CAS, Scopus and Google Scholar

- Research which is freely available for redistribution 